\title{
Male sexual functions and behaviors in the age of COVID-19: Evaluation of mid-term effects with online cross-sectional survey study
}

\author{
Erhan Ates, Hakan Gorkem Kazici, Ahmet Emre Yildiz, Saparali Sulaimanov, Arif Kol, Haluk Erol \\ Aydin Adnan Menderes University School of Medicine, Department of Urology, Aydin, Turkey.
}

\begin{abstract}
Summary Objective: To evaluate the long-term effects of the coronavirus disease 2019 (COVID-19) pandemic on sexual functions and behavior in men with heterosexual partners.

Materials and methods: A total of 602 participants completed an online questionnaire, shared via social networks, between November 20 and December 20, 2020. Pre-pandemic sexual intercourse frequency, International Erectile Dysfunction Index (IIEF-15) score, intravaginal ejaculatory latency time (IELT), premature ejaculation diagnostic tool (PEDT) score, and activities during sexual intercourse were compared to the ones during the pandemic. In addition, the effects of various variables on participants' sexual functions were evaluated and analyzed according to age groups.

Results: The mean number of weekly sexual intercourse during the pandemic was $1.7+1.7$, which was significantly lower than in the pre-pandemic period $(p<0.001)$. The ED score was significantly lower during the pandemic $(p<0.001)$ compared to the pre-pandemic period, however orgasmic function $(p=0.016)$, sexual intercourse satisfaction $(p<0.001)$, general satisfaction $(p<0.001)$, and PEDT scores $(p=0.004)$ were significantly higher. There was no significant difference in IELT before and during the pandemic ( $p=0.391)$. Full-time employment and low education level were risk factors for developing ED and PE. The negative affect of the pandemic on sexual life was most prominent in the $>65$ age group. Although kissing, oral and anal sex, and face-to-face sex positions decreased during the pandemic in all age groups, kissing and face-to-face sex positions remained the most preferred sexual behavior pattern $(p=0.002)$. There was no reduction in risky sexual behavior in the majority of the participants.

Conclusions: At the end of one year with COVID-19, a decrease in erectile function and an increase in PE incidence were observed in men. Despite this, there was an increase in sexual desire and satisfaction. Although there were some changes in sexual behavior, the majority of pre-pandemic habits continued.
\end{abstract}

KEY WORDS: Coronavirus disease 2019 (COVID-19); Erectile dysfunction; Premature ejaculation; Sexual behavior; Mid-term effect.

Submitted 1 March 2021; Accepted 21 April 2021

\section{INTRODUCTION}

Coronavirus disease 2019 (COVID-19) caused by the SARS CoV-2 virus first appeared in Wuhan, China, in December 2019 (1). It quickly spread across the globe, and finally the World Health Organization (WHO) declared it a pandemic on March 11, 2020 (2). The WHO generated a global epidemiological situation report based on the national data received from each country and Turkey was reported as having the highest number of cases in the European region (33\% of all cases in Europe, 194.476 new cases total, and 2.306 new cases per 1 million population) (3). The first COVID-19 case in Turkey was identified on the same day the WHO declared the global pandemic. Several measures limiting individual and social life were quickly implemented by the Turkish government. People have been living with these limitations, and the accompanying physical, psychological, economic, and social effects, for a long time.

It was inevitable that sexual health, defined by the WHO as the physical, emotional, mental, and social well-being of an individual, would also be affected during this period (4). Quarantine measures and some of the limitations in daily life imposed during the recent Severe Acute Respiratory Syndrome (SARS), H1N1 influenza, Middle East Respiratory Syndrome (MERS), and Ebola epidemics have been reported to negatively affect sexual life (5). The COVID-19 pandemic has significantly affected the quality of life, with negative effects on interpersonal relationships, community life, and sexual health (6). A few studies have evaluated the effects of the COVID-19 pandemic on sexual life during the first months of the pandemic; however they mostly focused on investigating sexual behaviors rather than sexual function, and presented various opinions $(7,8)$. The effects of this prolonged pandemic, which has reshaped all our lives, on sexual behavior patterns and sexual function are not yet known. In this study, we evaluated the medium-term effects of the COVID-19 pandemic on sexual function and behaviors in men with heterosexual partners.

\section{Materials AND METHOdS}

\section{Study design}

In this cross-sectional study, men were asked to complete a 34-item online questionnaire, which took approximately 20 minutes, consisting of multiple-choice and open-ended questions evaluating their sexual function and behaviors between November 06 and December 06, 2020. The questionnaire, which was created using a Turkish online survey

\footnotetext{
No conflict of interest declared.
} 
platform (http://www.surveey.com), was delivered to the participants online via social networks. The inclusion criteria of the study were being a heterosexual male older than 18 years, having an active sexual life that had continued for at least 6 months in the pre-pandemic period, no history of radical pelvic surgery, sexual dysfunction, or psychiatric disorder, and not having COVID-19. We evaluated men with heterosexual partners, because it has been reported that performing sexual activity with a partner provides higher sexual satisfaction than activity alone (9), and since the intravaginal ejaculation latency time (IELT) was used for evaluation of ejaculation function. Inclusion criteria were presented on the survey entry page on the website, and those who met these conditions and agreed to participate were asked to fill out the survey.
Sociodemographic data, medical history, and personal habits were investigated in the first part of the study. Age, education, marital status, work situation, income level during the pandemic, the number of individuals living at home during the pandemic, comorbidities, smoking, and alcohol use were evaluated. The second part of the study focused on evaluating changes in sexual behaviors such as the number of episodes of intercourse per week, IELT, sexual activities during intercourse, risky sexual activities, and changes in sexual attitudes such as the timing of intercourse during the day, during the pandemic, compared to the pre-pandemic period. In addition, the psychological effects of information about the pandemic obtained from news sources (television, radio, newspapers, magazines, and social media applications such as

Table 1.

Comparison of demographic data between group 1 and group 2.

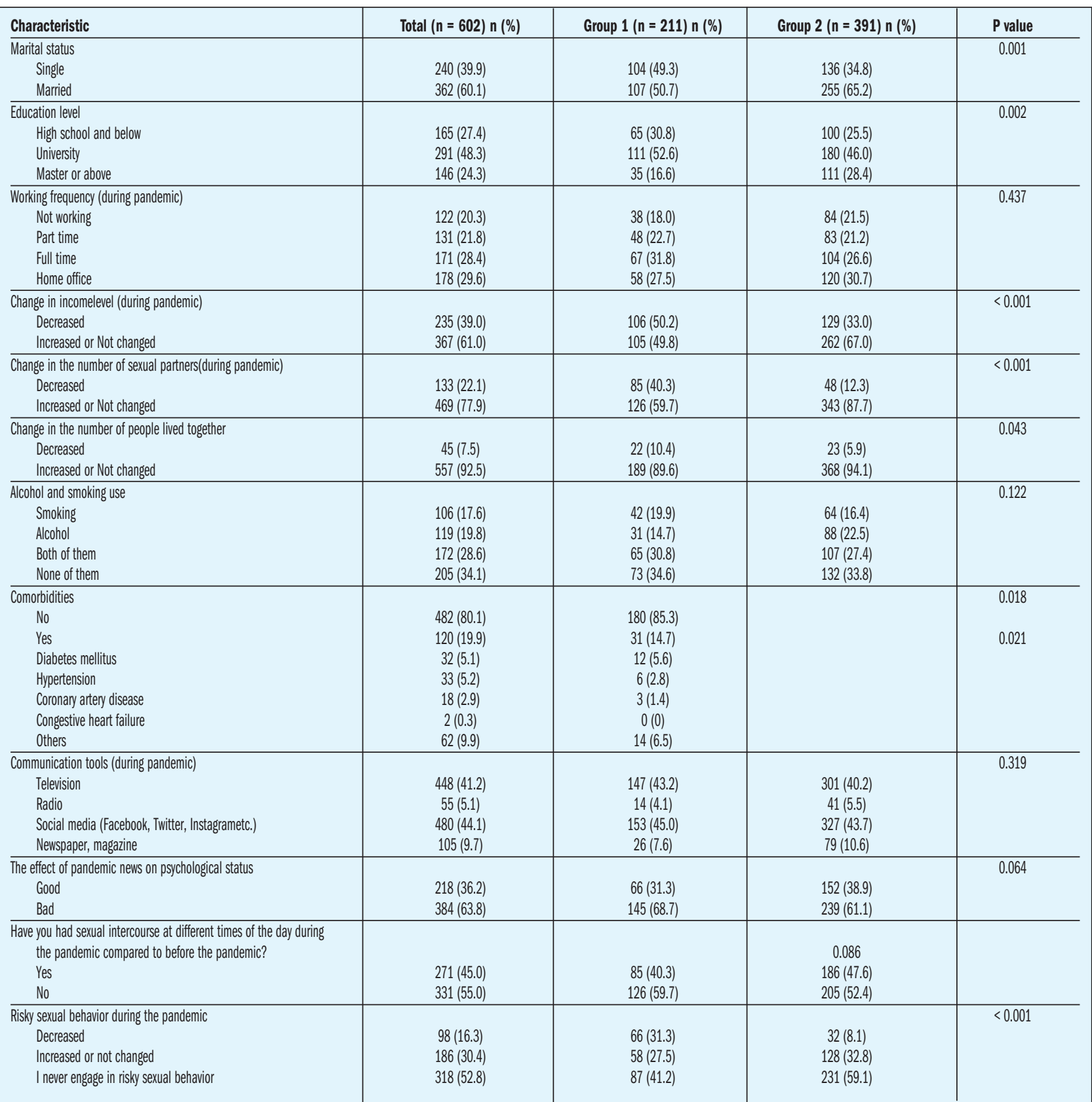


Facebook, Twitter, Instagram, and WhatsApp), and whether such news had a negative effect on their sexual activity during the pandemic were investigated. In the third part of the study, sexual functioning including erection and ejaculation were evaluated using internationally validated questionnaires.

This study complied with the relevant ethical regulations (institutional ethics committee protocol number: 2020/117). All participants reviewed and signed the informed consent page prior to filling out the online survey on the website.

\section{Evaluation of sexual function}

The International Index of Erectile Function (IIEF-15), which was translated and validated by the Turkish Andrology Association in 2002, was used to investigate five areas of male sexual function, including erectile function, orgasmic function, sexual desire, sexual satisfaction, and general satisfaction before and during the pandemic (10). In our study, participants with an IIEF score $<26$ were considered to have erectile dysfunction (ED), and those $\geq$ 26 were considered to have normal erectile function.

Participants were asked to choose their IELT from one of the following options: < $1 \mathrm{~min}, 1-3 \mathrm{~min}, 3-25 \mathrm{~min}$, and $\geq 25 \mathrm{~min}$. The premature ejaculation diagnostic tool (PEDT), a 5-item questionnaire that evaluates the control, frequency, minimum stimulation, distress, and interpersonal difficulties of ejaculation, the Turkish version of which has been validated, was used in the evaluation of premature ejaculation (PE) before and during the pandemic (11). In our study, participants with a PEDT score of $\geq 9$ were considered to have PE.

Participants who reported a decrease in the number of episodes of sexual intercourse per week during the pandemic were classified as Group 1, and those who reported an increase or no change as Group 2. In addition, participants were divided into three groups according to their ages: $<40$ years, $40-65$ years, and $\geq 65$ years.

The changes in these groups from before and during the pandemic were compared. Also, the characteristics of men who had normal erectile function before the pandemic, but had newly developed ED and PE during the pandemic, were evaluated by logistic regression analysis.

\section{Statistical analyses}

The survey data were evaluated using SPSS software (ver.21.0 for Windows; SPSS Inc, Chicago, IL, USA).

The compliance of continuous variables with normal distribution was investigated using visual (histogram and probability graphs) and analytical (Kolmogorov-Smirnov/ Shapiro-Wilk tests) methods. For the descriptive statistics, mean and standard deviation were used for data that fitted the normal distribution, and the median and minimummaximum for data that did not fit the normal distribution. Chi-square test was used to determine whether there was a difference between categorical variables. Student's t-test or one-way ANOVA were used to compare continuous variables with parametric properties in independent groups, and Mann-Whitney U Test or Kruskal-Wallis analysis of variance were used to compare continuous variables without parametric properties in independent groups. The T-test was used to compare continuous vari- ables with parametric properties in dependent groups, the Wilcoxon test was used to compare continuous variables with non-parametric properties in dependent groups, and McNemar's Chi-Square test was used to compare categorical variables in dependent groups. P value of $<0.05$ was considered statistically significant.

\section{RESULTS}

A total of 1.309 men participated in the study. The data of 602 participants who completed the questionnaire and met the inclusion criteria were evaluated. The response rate was $45.9 \%$. The mean age of all participants was 36.1 \pm 11.6 years. While the mean weekly frequency of sexual intercourse was $2.1 \pm 1.5$ in the pre-pandemic period, it was $1.7 \pm 1.7$ during the pandemic $(p<0.001)$. Of the participants, 35\% ( $\mathrm{n}=211)$ were in Group 1 and 65\% (n $=391$ ) in Group 2. The demographic characteristics of all participants and the distribution of these characteristics according to both groups are summarized in Table 1.

Erectile function score during the pandemic was significantly lower $(\mathrm{p}<0.001)$ than before the pandemic, while orgasmic function $(p=0.016)$, sexual intercourse satisfaction $(\mathrm{p}<0.001)$, general satisfaction $(\mathrm{p}<0.001)$, and PEDT $(p=0.004)$ scores were significantly higher (Table 2). All subdomains of IIEF-15 were significantly lower in Group 1 than in Group 2. However, the PEDT score was higher in Group 1, but not statistically significant $(p=0.055)$. No significant difference was found in self-reported IELT scores before and during the pandemic $(\mathrm{p}=0.391)$ (Figure 1$)$.

While a significant decrease was observed in sexual behaviors such as kissing, oral sex and face-to-face sex positions during the pandemic, a significant increase was observed in non-face-to-face positions (Table 3). During the pandemic, $45 \%(n=271)$ of participants had intercourse at times of the day that differed from their pre-pandemic routine habits. Among 284 who reported risky sexual behaviors such as sexual intercourse without condoms, multiple partners, intercourse with new acquaintances and/or sex workers in the pre-pandemic period, only 98 (34.5\%) reported a decrease in these behaviors during the pandemic.

Evaluation according to age groups indicated that all IIEF15 subdomains were significantly lower in the $\geq 65$ years age group compared to other groups during the pandemic. In addition, their PEDT scores were significantly higher than other age groups. In terms of sexual behaviors, kissing, and face-to-face sex positions decreased during the pandemic in all age groups but remained the most common sexual behavior pattern $(\mathrm{p}=0.002)$.

The data of 39 participants (8.8\%) who had normal erectile function (IIEF score $\geq 26$ ) before the pandemic, but developed ED of varying degrees (IIEF $<26$ ) during the pandemic, and 25 (5.3\%) participants who did not have PE complaints (PEDT score $<9$ ) before the pandemic, but were assessed as having PE during the pandemic (PEDT score $\geq 9$ ), were evaluated separately. When the factors affecting the development of ED and PE during the pandemic were examined with univariate analysis, ED was significantly more common in full-time workers (odds ratio: 5.011, 95\% confidence interval: 1.191-21.090, $p=0.028$, while PE was significantly more common in 


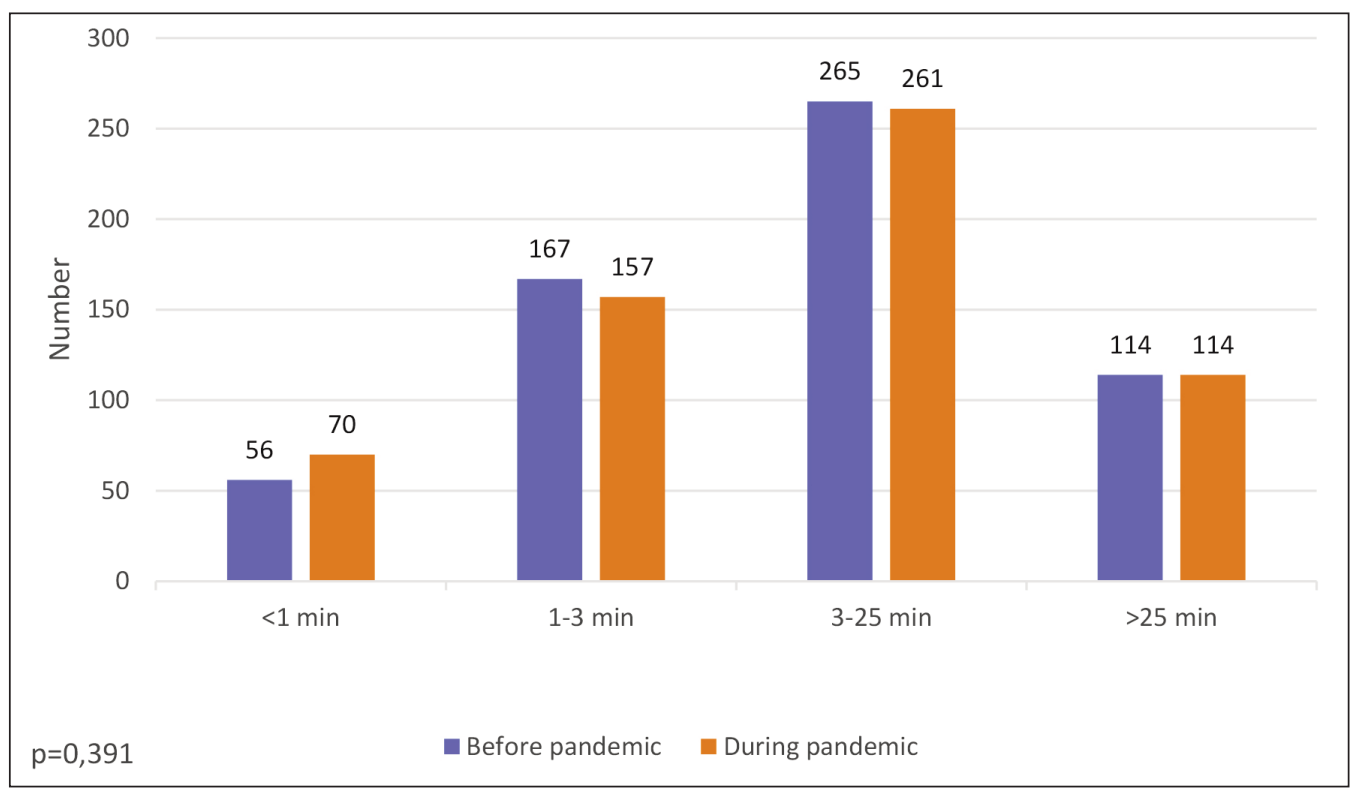

Figure 1

Self-reported IELT scores before and during the pandemic. men with a lower education level (odds ratio: $1.892,95 \%$ confidence interval: 0.708-5.056, $\mathrm{p}=0.040$ ) (Table 4). Most of respondents obtained information about the
COVID-19 pandemic through social media applications (Facebook, Twitter, Instagram, WhatsApp) (44.1\%) and television (41.2\%). Among the participants, 63.8\% stated

Table 2.

Comparison of male sexual functions according to pre-pandemic, post-pandemic, group 1 and group 2, and age groups.

\begin{tabular}{|c|c|c|c|c|c|c|}
\hline & \multicolumn{5}{|c|}{ International Index of Erectile Function-15 (IIEF-15) } & \multirow[t]{2}{*}{ Premature Ejaculation Diagnostic Tool (PEDT) } \\
\hline & $\begin{array}{l}\text { Erectile function } \\
(\max \text { score } 30)\end{array}$ & $\begin{array}{l}\text { Orgasmic function } \\
\text { (max. score 10) }\end{array}$ & $\begin{array}{c}\text { Sexual desire } \\
\text { (max. score 10) }\end{array}$ & $\begin{array}{l}\text { Intercourse satisfaction } \\
\text { (max. score 15) }\end{array}$ & $\begin{array}{l}\text { Overall satisfaction } \\
\text { (max. score 10) }\end{array}$ & \\
\hline $\begin{array}{l}\text { Before pandemic }(n=602) \\
\text { mean (SD) } \\
\text { median (min-max) }{ }^{*}\end{array}$ & $\begin{array}{l}26.9(5.2) \\
30(6-30)\end{array}$ & $\begin{array}{l}3.9(1.7) \\
4(2-10)\end{array}$ & $\begin{array}{l}3.7(1.7) \\
4(2-10)\end{array}$ & $\begin{array}{l}5.3(2.1) \\
5(0-12)\end{array}$ & $\begin{array}{l}4.0(2.0) \\
4(0-10)\end{array}$ & $\begin{array}{l}4.1(6.5) \\
0(0-20)\end{array}$ \\
\hline $\begin{array}{l}\text { During pandemic }(n=602) \\
\text { mean (SD) } \\
\text { median (min-max) }{ }^{*}\end{array}$ & $\begin{array}{l}26.5(6.0) \\
30(6-30)\end{array}$ & $\begin{array}{l}4.1(2.2) \\
4(2-10)\end{array}$ & $\begin{array}{l}3.9(2.0) \\
4(2-10)\end{array}$ & $\begin{array}{l}6.0(2.0) \\
6(0-11)\end{array}$ & $\begin{array}{l}4.9(2.2) \\
4(0-10)\end{array}$ & $\begin{array}{l}4.3(6.3) \\
0(0-20)\end{array}$ \\
\hline $\mathrm{P}$ value & $<0.001$ & 0.016 & 0.082 & $<0.001$ & $<0.001$ & 0.004 \\
\hline $\begin{array}{l}\text { Group } 1(\mathrm{n}=211) \\
\text { mean+SD } \\
\text { median }(\min -\mathrm{max})^{*}\end{array}$ & $\begin{array}{l}25.6(6.6) \\
30(6-30)\end{array}$ & $\begin{array}{l}4.0(2.2) \\
4(2-10)\end{array}$ & $\begin{array}{l}3.7(2.0) \\
3(2-10)\end{array}$ & $\begin{array}{l}5.9(1.7) \\
6(0-11)\end{array}$ & $\begin{array}{l}4.6(1.9) \\
4(0-10)\end{array}$ & $\begin{array}{l}4.9(6.5) \\
0(0-20)\end{array}$ \\
\hline $\begin{array}{l}\text { Group } 2(\mathrm{n}=391) \\
\text { mean+SD } \\
\text { median }(\text { min-max) }\end{array}$ & $\begin{array}{l}26.9(5.6) \\
30(6-30)\end{array}$ & $\begin{array}{l}4.4(2.1) \\
482-10)\end{array}$ & $\begin{array}{l}4.3(2.1) \\
4(2-10)\end{array}$ & $\begin{array}{l}6.2(2.4) \\
6(0-11)\end{array}$ & $\begin{array}{l}5.582 .5) \\
6(0-10)\end{array}$ & $\begin{array}{l}4.0(6.2) \\
0(0-20)\end{array}$ \\
\hline Pvalue & 0.008 & 0.005 & $<0.001$ & 0.043 & $<0.001$ & $0.055^{*}$ \\
\hline $\begin{array}{l}\text { Before pandemic }<40 \text { years }(n=436) \\
\text { mean+ }+S D \\
\text { median (min-max) }\end{array}$ & $\begin{array}{l}28.3(3.6) \\
30(8-30)\end{array}$ & $\begin{array}{c}3.9(1.7) \\
4(2-8)\end{array}$ & $\begin{array}{l}5.0(2.9) \\
5(2-10)\end{array}$ & $\begin{array}{c}7.2(1.8) \\
7(0-9)\end{array}$ & $\begin{array}{c}6.3(1.7) \\
6(0-9)\end{array}$ & $\begin{array}{l}3.5(6.3) \\
0(0-20)\end{array}$ \\
\hline $\begin{array}{l}0-65 \text { years }(n=145) \\
\text { mean+SD } \\
\text { median (min-max) }{ }^{*}\end{array}$ & $\begin{array}{l}24.7(5.7) \\
26(8-30)\end{array}$ & $\begin{array}{c}4.0(1.8) \\
4(2-8)\end{array}$ & $\begin{array}{c}4.0(1.7) \\
4(2-8)\end{array}$ & $\begin{array}{l}5.6(1.9) \\
5(0-11)\end{array}$ & $\begin{array}{l}4.3(1.8) \\
4(0-10)\end{array}$ & $\begin{array}{l}5.0(6.5) \\
2(0-20)\end{array}$ \\
\hline $\begin{array}{l}>65 \text { years }(n=21) \\
\text { mean+SD } \\
\text { median }(\text { min-max })^{*}\end{array}$ & $\begin{array}{l}13.9(6.6) \\
15(6-30)\end{array}$ & $\begin{array}{l}3.9(1.7) \\
4(2-10)\end{array}$ & $\begin{array}{c}3.6(1.6) \\
4(2-8)\end{array}$ & $\begin{array}{l}5.2(2.2) \\
5(0-12)\end{array}$ & $\begin{array}{l}3.8(1.9) \\
4(0-10)\end{array}$ & $\begin{array}{l}8.3(7.6) \\
12(0-19)\end{array}$ \\
\hline Pvalue & $<0.001$ & 0.993 & 0.006 & $<0.001$ & $<0.001$ & $<0.001$ \\
\hline $\begin{array}{l}\text { During pandemic }<40 \text { years }(n=436) \\
\text { mean+SD } \\
\text { median (min-max) }{ }^{*}\end{array}$ & $\begin{array}{l}28.0(4.4) \\
30(6-30)\end{array}$ & $\begin{array}{c}4.9(2.2) \\
4(2-8)\end{array}$ & $\begin{array}{l}6.0(2.5) \\
7(2-10)\end{array}$ & $\begin{array}{l}7.4(1.2) \\
7(4-10)\end{array}$ & $\begin{array}{l}6.7(1.2) \\
6(4-10)\end{array}$ & $\begin{array}{l}3.7(6.1) \\
0(0-20)\end{array}$ \\
\hline $\begin{array}{l}0-65 \text { years }(n=145) \\
\text { mean+SD } \\
\text { median (min-max) }{ }^{*}\end{array}$ & $\begin{array}{l}23.7(6.9) \\
25(6-30)\end{array}$ & $\begin{array}{l}4.5(2.2) \\
4(2-10)\end{array}$ & $\begin{array}{l}4.7(2.1) \\
4(2-10)\end{array}$ & $\begin{array}{l}6.1(2.1) \\
6(0-11)\end{array}$ & $\begin{array}{l}5.0(2.3) \\
4(0-10)\end{array}$ & $\begin{array}{l}5.6(6.2) \\
4(0-20)\end{array}$ \\
\hline $\begin{array}{l}>65 \text { years }(n=21) \\
\text { mean+SD } \\
\text { median }(\min -\mathrm{max}){ }^{*}\end{array}$ & $\begin{array}{l}14.4(6.5) \\
15(6-30)\end{array}$ & $\begin{array}{l}4.0(2.1) \\
4(2-10)\end{array}$ & $\begin{array}{l}3.5(1.8) \\
3(2-10)\end{array}$ & $\begin{array}{l}5.9(1.9) \\
6(0-11)\end{array}$ & $\begin{array}{l}4.8(2.1) \\
4(0-11)\end{array}$ & $\begin{array}{l}9.6(6.8) \\
12(0-19)\end{array}$ \\
\hline Pvalue & $<0.001$ & 0.007 & $<0.001$ & $<0.001$ & $<0.001$ & $<0.001$ \\
\hline
\end{tabular}


Table 3.

Comparison of sexual intercourse and sexual activities by pre-pandemic and post-pandemic, group 1 and group 2, and age groups.

\begin{tabular}{|c|c|c|c|c|c|c|}
\hline & \multirow{2}{*}{$\begin{array}{l}\text { Number of sexual intercoursein a week, } \\
\text { mean (SD) }\end{array}$} & \multirow[b]{2}{*}{ Kissing } & \multirow[b]{2}{*}{ Oral sex } & \multicolumn{2}{|c|}{ Sexual activities, $\mathbf{n}(\%)$} & \multirow[b]{2}{*}{$\begin{array}{c}\text { Nonface-to-face } \\
\text { Sex positions (e.g. doggystyle) }\end{array}$} \\
\hline & & & & Anal sex & $\begin{array}{c}\text { Face-to-face } \\
\text { Sex positions (e.g. missionary) }\end{array}$ & \\
\hline Before pandemic $(n=602)$ & $2.1(1.5)$ & $488(29.9)$ & $246(15.1)$ & $42(2.6)$ & $522(32.0)$ & $332(20.4)$ \\
\hline During pandemic $(n=602)$ & $1.7(1.7)$ & $402(28.3)$ & $200(14.1)$ & $33(2.3)$ & $429(30.2)$ & $357(25.1)$ \\
\hline Pvalue & $<0.001$ & $<0.001$ & $<0.001$ & 0.064 & $<0.001$ & 0.023 \\
\hline Group $1(n=211)$ & $1.0(1.0)$ & $130(28.0)$ & $73(15.7)$ & $12(2.6)$ & $129(27.8)$ & $120(25.9)$ \\
\hline Group $2(n=391)$ & $2.1(1.9)$ & 272 (28.4) & $127(13.3)$ & $21(2.2)$ & $300(31.3)$ & $237(24.8)$ \\
\hline Pvalue & $<0.001$ & & & & 554 & \\
\hline \multicolumn{7}{|l|}{ Age (Before pandemic), year } \\
\hline$<40$ years $(n=436)$ & $1.8(1.9)$ & $279(27.5)$ & $168(16.6)$ & $23(2.3)$ & $289(28.5)$ & $255(25.1)$ \\
\hline $40-65$ years $(n=145)$ & $1.6(1.1)$ & $104(29.1)$ & $31.0(8.7)$ & $10(2.8)$ & $121(33.8)$ & $92(25.7)$ \\
\hline$>65$ years $(n=21)$ & $0.8(0.5)$ & $19(38.8)$ & $1(2.0)$ & $0(0.0)$ & $19(38.8)$ & $10(20.4)$ \\
\hline Pvalue & 0.017 & & & & 002 & \\
\hline
\end{tabular}

\section{Table 4.}

Univariate analysis of erectile dysfunction and premature ejaculation.

\begin{tabular}{|c|c|c|c|c|c|c|}
\hline & \multicolumn{6}{|c|}{ Univariate analysis } \\
\hline & \multicolumn{3}{|c|}{ Erectile dysfunction } & \multicolumn{3}{|c|}{ Premature ejaculation } \\
\hline & $\mathrm{OR}$ & $\mathbf{p}$ & $\% 95 \mathrm{Cl}$ & OR & $\mathbf{p}$ & $\% 95 \mathrm{Cl}$ \\
\hline$\overline{\text { Age }}$ & 1.002 & 0.903 & $0.974-1.030$ & 0.995 & 0.833 & $0.953-1.039$ \\
\hline Education level & 1.191 & 0.627 & $0.589-2.410$ & 1.892 & 0.040 & $0.708-5.056$ \\
\hline Working frequency (during pandemic) & 5.011 & 0.028 & $1.191-21.090$ & 1.192 & 0.786 & $0.337-4.214$ \\
\hline Change in income level (during pandemic) & 0.732 & 0.348 & $0.381-1.404$ & 0.438 & 0.098 & $0.164-1.166$ \\
\hline Marital status & 0.835 & 0.601 & $0.425-1.641$ & 1.352 & 0.540 & $0.514-3.556$ \\
\hline Change in the number of sexual partners (during pandemic) & 0.542 & 0.084 & $0.270-1.086$ & 2.164 & 0.309 & $0.489-9.585$ \\
\hline Change in the number of people lived together & 0.967 & 0.957 & $0.286-3.273$ & 1.301 & 0.801 & $0.169-10.043$ \\
\hline Comorbidities & 3.148 & 0.060 & $0.953-10.402$ & 0.587 & 0.326 & $0.203-1.700$ \\
\hline The effect of pandemic news on psychological status & 0.589 & 0.160 & $0.281-1.232$ & 1.241 & 0.666 & $0.465-3.308$ \\
\hline
\end{tabular}

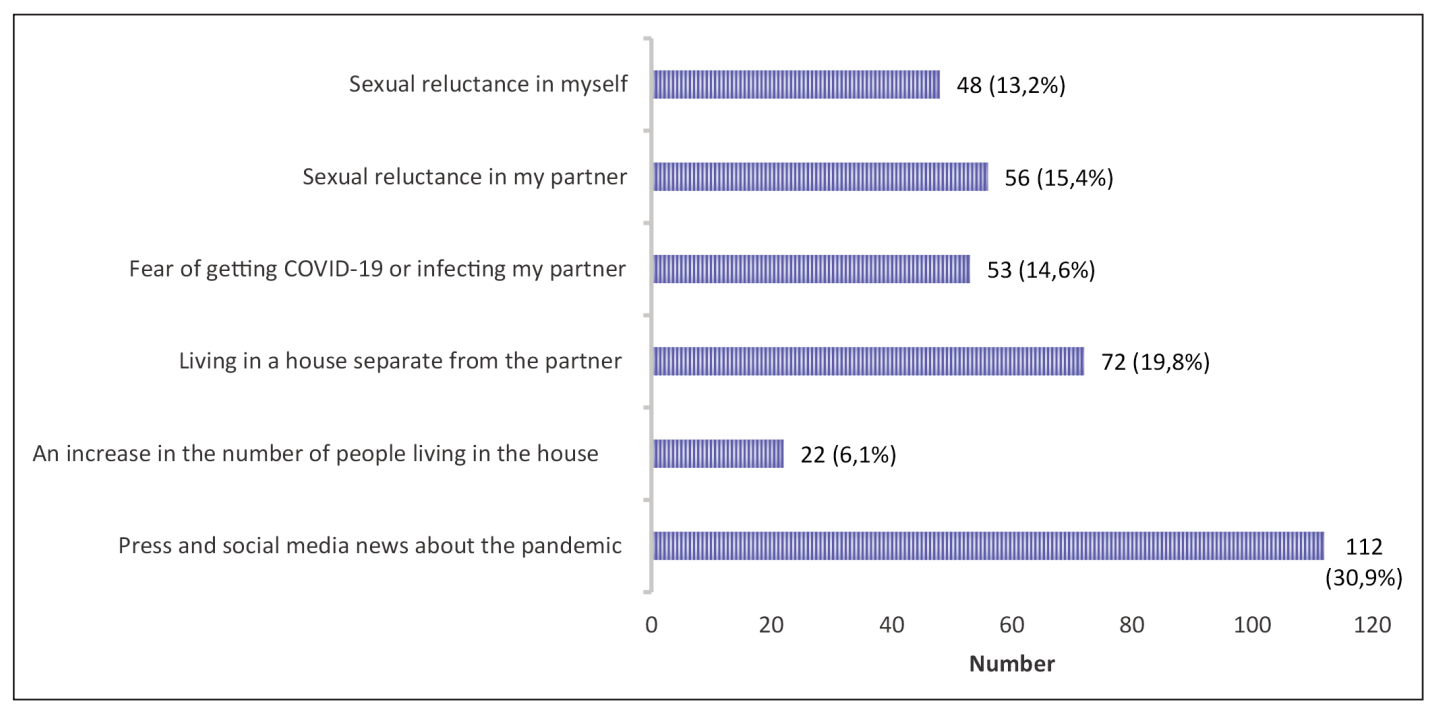

Figure 2.

The reasons reported by the participants for the decrease in sexual activities during the pandemic. that they were psychologically negatively affected by this information, and $30.9 \%$ attributed their decrease in frequency of sexual intercourse during the pandemic to the news obtained from the press and social media (Figure 2).

\section{Discussion}

This is the first study to evaluate medium-term effects of the COVID-19 pandemic on sexual function and behavior in men. In this study, as in many reports in the litera- ture, the number of episodes of sexual intercourse was taken as the basis for sexual health evaluation. Although there are studies defending the contrary (12), one of the main factors determining sexual satisfaction remains the frequency of sexual intercourse (13). Sexual satisfaction is both the result and indicator of a healthy sex life (14).

An online survey study conducted in some Southeast Asian countries involving participants with a high level of education, reported that there was no significant difference in the frequency of sexual intercourse during the 
COVID-19 pandemic compared to the pre-pandemic time (15). Li et al. (8) found that during the pandemic, $37 \%$ of respondents experienced a decrease in the frequency of sexual intercourse and 25\% in sexual desire. These reductions were greater in men than in women, and a decrease in sexual satisfaction was found in 32\% of the men. Jacob et al. (7) found that young age, male gender, being married, and consuming alcohol were associated with increased sexual activity. An increased number of days spent in self-isolation also positively affected the frequency of sexual intercourse.

Studies reporting a decrease in the frequency of sexual desire and intercourse concluded that limited living space, prolonged decrease in domestic privacy, increase in differences of opinion between the spouses, and exacerbation of previous conflicts were reasons for the changes in sexual behavior. In addition, the effects of various pandemic-related stress factors, together with anxiety and depression caused by economic deterioration have also been reported (16). Although there are conflicting reports, most studies reported that the severity of anxiety and depression was correlated with the loss of sexual desire (17). In our study, although there was a decrease in the frequency of sexual intercourse, there was an increase in sexual desire and satisfaction. Various physical factors such as the requirement to live apart from their partner, and staying away from their partner due to fear of getting sick may have affected the frequency of sexual intercourse during this period (18). However, the increase in sexual desire may be a result of the individual's internal struggle in dealing with the long-term negative psychological factors. Mollaioli et al. (19) reported that all types of sexual activity have protective effects against anxiety and mood disorders related to quarantine in both sexes. However, it is not necessary to have a lot of sexual intercourse for sexual health and satisfaction (12). Although there is no evidence that COVID-19 is transmitted through sexual intercourse, the close contact of partners due to the nature of sex creates a potential risk for SARS-CoV-2 transmission through respiration and saliva. This, in addition to other factors, can lead to avoidance of sexual activity during the pandemic despite a healthy partner (20). Even if intercourse is not avoided, activity preferences during intercourse may be affected. Culha et al. (21) reported that foreplay, kissing, oral, and anal sex were less common and that couples preferred non-face-to-face sexual positions during the COVID-19 pandemic. However, Baran and Aykac (22) reported that the vast majority of couples did not fear COVID-19 transmission during sex, and married couples had the least amount of fear. In our study, only $14.6 \%$ of men stated that their sex life was negatively affected due to the fear of infecting themselves or their partner. Face-to-face positions such as the missionary position in vaginal intercourse as well as kissing, oral, and anal sex decreased compared to the pre-pandemic period, but face-to-face positions remained the preferred type of sexual behavior during the pandemic. In addition, we found that only $34.5 \%$ of men who reported risky sexual activities in terms of transmission such as sexual activity without a condom, multiple partners, and intercourse with new acquaintances or sex workers during the pre-pandemic period reduced such activities during the pandemic. However, a decrease in risky sexual behaviors has been reported in the early stages of the pandemic (8).

Erectile function was evaluated with international erectile function indices (IIEF-5, IIEF-15), and a decrease was found in the early stages of COVID-19 compared to the pre-pandemic period (22). Fang et al. (23) stated that men reported worsening of erectile function and ejaculation control ability during the pandemic. In that study, it was observed that $31.9 \%$ of participants had a decrease in their IIEF-5 scores, and $17.9 \%$ had an increase in their PEDT scores. In our study, the erection score was significantly lower but all other subdomains of IIEF-15 were significantly higher during the pandemic. The number of men who reported their IELT as $<1$ minute during the pandemic was higher than before the pandemic. In addition, there was a significant increase in the PEDT score compared to the pre-pandemic period. In the European Urology Guideline, it has been reported that PE is affected by low education level, absence of physical activity, and religious beliefs (a majority of Muslim countries have higher levels of PE) (24). Stress and limitation of movement in a country with a majority Muslim population such as Turkey might therefore be expected to cause an increase in PE. However, we did not find any risk factor other than a low education level in the univariate analysis of respondents with newly developed PE during the pandemic.

With advanced age, low testosterone levels in men increase the loss of libido and negatively affect sexual behavior, thus reducing the quality of sexual life (25).

It is not surprising that erectile function also decreases due to androgen deficiency and increased stress factors. We found that erectile function and sexual desire were lower in the older age group. When we evaluated PE according to age groups, we found that during the pandemic PEDT scores were higher in men $\geq 65$ years of age. Our study had some limitations. The data were selfreported. Requesting information from the pre-pandemic period may have created memory difficulties for participants and introduced bias. Other than those of the international questionnaires, our questions were non-validated. In addition, because we wanted to keep the number of questions low to make participation easier, we did not evaluate their current anxiety and/or depression status with approved questionnaires. We only included participants who did not have a known psychiatric problem. However, participants reported that they were psychologically negatively affected by news about COVID-19 and that this affected their sexual life the most. This can be considered a separate limitation. Society's perception of sexuality is effective in shaping individual sexual behavior. This may limit generalization of our results. We believe that the results of our study, conducted in Turkey, where the culture is a blend of the values of both western and eastern civilizations, can contribute to knowledge about changes in sexual function and behavior during the COVID-19 pandemic.

\section{Conclusions}

It has been one year since the start of the COVID-19 pandemic. In our study of men in Turkey, frequency of sex- 
ual intercourse and erectile function have decreased, although sexual desire and sexual satisfaction have both increased. In addition, complaints of PE have increased. In terms of sexual behavior, pre-pandemic habits have continued, including engaging in risky sexual behavior.

\section{REFERENCES}

1. Zhu $N$, Zhang $D$, Wang $W$, et al. A novel Coronavirus from patients with pneumonia in China. N Engl J Med. 2019; 382:727-33.

2. World Health Organization, (2020). (March 11, 2020). DirectorGeneral's opening remarks at the media briefing on COVID-19-11 March 2020. Retrieved from https://www.who.int/dg/speeches/detail/ who-director-general-s-opening-remarks-at-the-media-briefing-oncovid-19--11-march-2020.

3. World Health Organization, (2020). (December 22, 2020). Coronavirus disease (COVID-19) Weekly Epidemiological Update and Weekly Operational Update-22 December 2020. Retrieved from https://www.who.int/publications/m/item/weekly-epidemiologicalupdate---22-december-2020.

4. Glasier A, Gülmezoglu AM, Schmid GP, et al. Sexual and reproductive health: a matter of life and death. Lancet. 2006; 368:1595-607.

5. Brook SK, Webster RK, Smith LE, et al. The psychological impact of quarantine and how to reduce it: rapid review of the evidence. Lancet 2020; 395:912-20.

6. Maretti C, Privitera S, Arcaniolo D, et al. COVID-19 pandemic and its implications on sexual life: Recommendations from the Italian Society of Andrology. Arch Ital Urol Androl. 2020; 92:73-7.

7. Jacob L, Smith L, Butler L, et al. Challenges in the practice of sexual medicine in the time of COVID-19 in the United Kingdom. J Sex Med. 2020; 17:1229-36.

8. Li W, Li G, Xin C, et al. Challenges in the practice of sexual medicine in the time of COVID-19 in China. J Sex Med. 2020; 17:1225-8.

9. Brod S, Costa RM. Satisfaction (sexual, life, relationship, and mental health) is associated directly with penile-vaginal intercourse, but inversely with other sexual behavior frequencies. J Sex Med. 2009; 6:1947-1954.

10. Akkus E, Kadioglu A, Esen A, et al. Prevalence and correlates of erectile dysfunction in Turkey: a population-based study. Eur Urol. 2002; 41:298-304

11. Serefoglu EC, Cimen HI, Ozdemir AT, et al. Turkish validation of the premature ejaculation diagnostic tool and its association with intravaginal ejaculatory latency time. Int J Impot Res. 2009; 21:13944.

12. Muise A, Schimmack U, Impett EA. Sexual frequency predicts greater well-being, but more is not always better. Soc Psychol Personal Sci. 2015; 7:295-302.

13. Gillespie BJ. Correlates of sex frequency and sexual satisfaction among partnered older adults. J Sex Marital Ther. 2017; 43:403-23.

14. Palha-Fernandes E, Alves P, Lourenço M. Sexual satisfaction determinants and its relation with perfectionism: A cross-sectional study in an academic community. Sexual and Relationship Therapy, DOI: $10.1080 / 14681994.2019 .1677884$.

15. Arafat SMY, Alradie-Mohamed A, Kar SK, et al. Does COVID19 pandemic affect sexual behaviour? A cross-sectional, crossnational online survey. Psychiatry Res. 2020; 289:113050.

16. Ko NY, Lu WH, Chen YL, et al. Changes in sex life among people in Taiwan during the COVID-19 pandemic: the roles of risk per- ception, general anxiety, and demographic characteristics. Int J Environ Res Public Health. 2020; 17:5822.

17. Beutel ME, Burghardt J, Tibubos AN, et al. Declining sexual activity and desire in men-findings from representative German surveys, 2005 and 2016. J Sex Med. 2018; 15:750-6.

18. Döring N. How Is the COVID-19 pandemic affecting our sexualities? An overview of the current media narratives and research hypotheses. Arch Sex Behav. 2020; 49:2765-78.

19. Mollaioli D, Sansone A, Ciocca G, et al. Benefits of sexual activity on psychological, relational, and sexual health during the COVID-19 breakout. J Sex Med. 2021; 18:35-49.

20. Scorzolini L, Corpolongo A, Castilletti C, et al. Comment on the potential risks of sexual and vertical transmission of COVID-19. Clin Infect Dis. 2020; 71:2298.

21. Culha MG, Demir O, Sahin O, Altunrende F. Sexual attitudes of healthcare professionals during the COVID-19 outbreak. Int J Impot Res. 2021; 33:102-9.

22. Baran O, Aykac A. The effect of fear of covid-19 transmission on male sexual behaviour: A cross-sectional survey study. Int J Clin Pract. 2021; 75:e13889.

23. Fang D, Peng J, Liao S, et al. An online questionnaire survey on the sexual life and sexual function of Chinese adult men during the Coronavirus disease 2019 Epidemic. Sex Med. 2021; 9:100293.

24. Salonia A, Bettocchi C, Carvalho J, et al. Sexual and reproductive health. EAU Guidelines 2020. Edn. Presented at the EAU Annual Congress Amsterdam 2020. ISBN 978-94-92671-07-3.

25. Forbes MK, Eaton NR, Krueger RF. Sexual quality of life and aging: a prospective study of a nationally representative sample. J Sex Res. 2017; 54:137-48.

\section{Correspondence}

Erhan Ates, Associate Professor of Urology (Corresponding Author) drerhanates@yahoo.com

Hakan Gorkem Kazici, MD hgkazici@yahoo.com

Ahmet Emre Yildiz, MD aemreyildiz@gmail.com Saparali Sulaimanov, MD sulaimanovsaparali@gmail.com

Arif Kol, Assistant Professor of Urology

drarifkol@hotmail.com

Haluk Erol, Professor of Urology

halukerol@yahoo.com

Department of Urology, Aydin Adnan Menderes University School of Medicine, 09010, Aydin (Turkey) 